\title{
Les ambassadeurs suisses à Paris en 1663. Usages protocolaires : entre tradition et politique
}

\section{Claire-Marie Lomenech}

\section{(2) OpenEdition}

\section{Journals}

Édition électronique

URL : http://journals.openedition.org/edl/262

DOI : $10.4000 /$ edl. 262

ISSN : 2296-5084

Éditeur

Université de Lausanne

\section{Édition imprimée}

Date de publication : 15 septembre 2010

Pagination : 155-162

ISBN : 978-2-940331-23-9

ISSN : 0014-2026

Référence électronique

Claire-Marie Lomenech, « Les ambassadeurs suisses à Paris en 1663. Usages protocolaires : entre tradition et politique », Études de lettres [En ligne], 3 | 2010, mis en ligne le 15 septembre 2013, consulté le 22 décembre 2020. URL : http://journals.openedition.org/edl/262 ; DOl : https://doi.org/10.4000/ edl.262 


\section{LES AMBASSADEURS SUISSES À PARIS EN 1663 USAGES PROTOCOLAIRES : ENTRE TRADITION ET POLITIQUE}

Louis XIV invite les ambassadeurs des cantons suisses à jurer le renouvellement d'alliance le 18 novembre 1663 à Paris. L'étude du protocole en vigueur lors de cette cérémonie permettra une meilleure compréhension du rôle des ambassadeurs du Corps helvétique dans une mise en scène savamment orchestrée.

Les relations diplomatiques entre la France et les cantons suisses sous l'Ancien Régime ont été retracées principalement par deux historiens, Edouard Rott ${ }^{1}$ et Georges Livet ${ }^{2}$. Hormis quelques chapitres dans leurs ouvrages ou dans des publications plus récentes ${ }^{3}$, le renouvellement de l'alliance entre la France et les Suisses de 1663 n'a jamais fait l'objet d'une étude en soi. Quant aux articles de Sigrid Palmmert ${ }^{4}$, qui s'intéressent à l'histoire de l'art et à celle de la représentation, ils font seuls exception à ce que nous venons de dire.

Notre travail, inscrit dans le cadre d'un mémoire de maîtrise, s'appliquera à combler une lacune dans la perception française du protocole diplomatique. Nous analyserons les correspondances et les mémoires préparant ou relatant la prestation solennelle du serment à Notre-Dame de Paris, le 18 novembre 1663, afin de comprendre l'importance des

I. E. Rott, Histoire de la représentation diplomatique de la France auprès des cantons suisses, de leurs alliés et de leurs confédérés.

2. G. Livet, Recueil des instructions aux ambassadeurs et ministres de France.

3. Th. Maissen, Die Geburt der Republic; G. P. Marchal, Schweizer Gebrauchsgeschichte.

4. S. Palmmert, "Kleider machen Leute, Königen machen Mode»; Id., "Der Allianzteppich und die Fragen von Selbstdarstellung, Repräsentation und Rezeption». 
conventions cérémonielles dans les enjeux de représentation des Etats. Cette étude mettra aussi en lumière l'identité du personnel diplomatique helvétique au XVII e siècle: qui traite avec les puissances étrangères (principalement l'Espagne-Autriche et la France) et d'où ces diplomates sont-ils originaires?

En 1661, Charles Le Brun est nommé premier peintre de Louis XIV. Comme directeur de la Manufacture des Gobelins, il occupe un poste clé pour la diffusion de l'image du Roi-Soleil. C'est ainsi que, supervisé par la Petite Académie 5 , il dessine les cartons de l' "Histoire du Roy", une suite de 17 tapisseries exposant et glorifiant des scènes civiles, militaires ou diplomatiques. L'une d'entre elles retiendra particulièrement notre attention: la tapisserie du Renouvellement d'alliance entre la France et les Suisses fait dans l'Eglise Notre-Dame de Paris par le roi Louis XIV et les ambassadeurs des 13 cantons et leurs alliés, le 18 novembre 1663. Résultat de plus de quinze années de négociations entre l'ambassadeur de Sa Majesté et les Confédérés, l'alliance ici représentée est conclue à Soleure le 24 septembre 1663 avant d'être jurée à Paris deux mois plus tard. Les délégués helvétiques en partance pour la capitale française sont nombreux. Penchons-nous sur ceux qui représentent les intérêts de la Suisse.

Chaque canton désigne deux délégués pour ce voyage et les co-alliés un seul. Ainsi le groupe est constitué de trente-six ambassadeurs, parmi lesquels Johann Heinrich Waser, le bourgmestre de la ville de Zurich, nommé président de la délégation. Berne désigne son avoyer, Anthoni von Graffenried; Bâle, le bourgmestre Johann Rudolph Wettstein; Schwyz, le landamman Wolffgang Dietrich Reding. De Lucerne, sont aussi envoyés Christophe Pfyffer, l'avoyer de la ville et seigneur d'Altishofen, Fidèle vom Thurm, seigneur d'Eppenberg et de Buchwyl, de l'abbaye de Saint-Gall et trente autres. Ce sont donc pour la plupart des landammans en titre, ou anciens. Cela met-il en lumière le fait qu'en Suisse comme en France ${ }^{6}$, la profession de diplomate tend à se spécialiser? Dans son récit publié en $1664^{7}$ déjà, le secrétaire général de l'ambassade Johann Georg Wagner dresse la liste du personnel diplomatique

5. Future "Académie des Inscriptions et des Médailles».

6. L. Bély, «Souveraineté et souverains: la question du cérémonial dans les relations internationales à l'époque moderne», p. 42.

7. J. G. Wagner, Parisische Reyss, Handlung und Pundtschwur. 
$\geq 2$

$\therefore \sqrt{2}$

3.

थै

辛芒 
où on compte un secrétaire interprète, Jean Philippe Vigier, et d'autres secrétaires personnels, huissiers, domestiques, parents des ambassadeurs ou conducteurs de chevaux, qui portent le nombre des envoyés à deux cent vingt-sept.

Sur la tapisserie de l'alliance, on reconnaît deux personnages parmi les Suisses, tous vêtus de noir, nu-tête et barbus: Waser, et derrière lui Graffenried. Ces deux personnages sont mis en avant par rapport aux autres ambassadeurs parce que Waser est le représentant de Zurich, le canton qui "a obtenu le premier rang en tant que Vorort»" ${ }^{8}$ et Graffenried celui de Berne, second canton dans l'ordre diplomatique mais non moins puissant. Waser se tient en face du roi, une main sur l'Evangile, jurant l'alliance avec Louis XIV. Le Brun condense le fil narratif des serments successifs en une seule "prise de vue", afin d'en renforcer la signification et la solennité. En réalité, les ambassadeurs suisses jurent l'alliance les uns après les autres, avant que le roi prête serment à son tour en disant: "Et moi aussi, je jure et promets" ${ }^{9}$. Les visages des Confédérés semblent empreints de consternation et leur attitude contraste avec la gestuelle des courtisans français. Les négociations avec l'ambassadeur français Jean de la Barde ont en effet été longues et âpres, pour un résultat insatisfaisant puisque de fait, il place le Corps Helvétique sous la dépendance de la France. Les cantons suisses connaissent entre eux des mésententes. Les catholiques sont les plus prompts à signer, puisqu'ils renouvellent l'alliance en 1653. Bâle et Zurich, villes protestantes, sont fermement opposées à l'alliance avec la France, pour des raisons confessionnelles et financières, la France ayant beaucoup de retard dans le paiement de ses dettes. Lalliance avec les cantons évangéliques se fait finalement en 1658. Il reste à trouver un accord commun. Après cinq nouvelles années de négociations, l'alliance générale est signée à Soleure: à partir de ce moment-là, les Suisses n'ont plus aucun moyen de pression. Louis XIV se sachant en position de force n'hésite pas à refuser poliment leurs demandes en matière de cérémonial, dernier domaine où réside un véritable enjeu.

A leur retour au pays, on reprochera aux Confédérés leur manque de fidélité aux instructions reçues. Ils semblent aussi avoir été trop diligents à accepter les traitements du roi, artifices de Louis XIV afin de les lier et

8. G. Poisson, «La République de Berne vue par les diplomates français», p. 499.

9. "Relation du Cérémonial pour le renouvellement d'alliance», fol. 474. 
de faire taire leurs requêtes et plaintes ${ }^{10}$. Ont-ils été aveuglés par tant de luxe et de faste? Les grands moyens sont en effet mis en ouvre. Durant leur semaine à Paris, les ambassadeurs sont accueillis chez les plus grands princes lors de réceptions soigneusement planifiées par le roi lui-même. Si les alliances franco-suisses ont été jusqu'alors source d'intérêts pour les deux pays, 1663 marque un tournant dans cette dynamique. Les ambassadeurs suisses n'ont pas su éviter un traité qui lie leur pays de manière plus étroite que jamais à la France.

Ce renouvellement d'alliance est l'objet d'une riche correspondance entre Louis XIV et son ambassadeur à Soleure: la durée du séjour des diplomates suisses à Paris est d'environ trois semaines, chaque journée faisant l'objet d'une préparation minutieuse par le roi et ses ministres. A Neuchâtel, le fonds Rott bénéficie d'une documentation abondante parmi laquelle on compte ces lettres (entre la cour, l'ambassade de France à Soleure et les cantons suisses) ainsi que de nombreux mémoires. Nous possédons aussi trois témoignages contemporains des faits: le récit que Monsieur de Berlize, conducteur des ambassadeurs, fait de la semaine qui précède la cérémonie ${ }^{11}$, celui du fils d'André Lefèvre d'Ormesson qui officia comme chancelier ${ }^{12}$, ainsi que les mémoires de l'ambassadeur suisse Waser ${ }^{13}$. Nous travaillerons donc principalement sur deux types de documents: la correspondance et les mémoires, officiels et personnels. Les mémoires de Waser permettront particulièrement de comprendre la conception qu'il a de sa propre fonction et, confrontés aux documents français, mettront davantage en évidence les différences de culture entre les deux modèles politiques que sont la monarchie et la république, à travers la représentation des Etats.

\section{Claire-Marie LOMENEcH}

Université de Lausanne

Io. G. P. Marchal, «Le rôle de la représentation symbolique dans les représentations diplomatiques», p. 203.

II. Favre de Berlize, Mémoires de Monsieur de Berlize, Introducteur des Princes Etrangers et Ambassadeurs a la Cour de France depuis l'an 1639 jusqu'en 1663.

I2. O. Lefèvre d'Ormesson, Journal d'Olivier Lefèvre d'Ormesson et extraits des mémoires d'André Lefèvre d'Ormesson.

I3. J. H. Waser, Beschrybung dess Bundt Schwurs zwüschent König Ludwig XIV. zu Frankrych und Navarra und den XIII Orten auch etlichen Zugewandten loblicher Eydgnoschafft. Beschehen zu Paris im Novembri anno 1663. J. H. Waser. 


\section{BIBLIOGRAPHIE}

Textes

"Relation du Cérémonial pour le renouvellement d'alliance», in Ambassades en Suisse et aux Grisons. Cérémonial 1565-1750, vol. VII 11/1, BPU Neuchâtel, Fonds Edouard Rott, fol. 474.

Favre de Berlize, Mémoires de Monsieur de Berlize, Introducteur des Princes Etrangers et Ambassadeurs a la Cour de France depuis l'an 1639 jusquien 1663, transcrits par Patricia Baertsen, mémoire de maîtrise sous la direction de Lucien Bély, Université de Lille III Charles de Gaulle, septembre 1991.

Lefèvre d'Ormesson, Olivier, Journal d'Olivier Lefèvre d'Ormesson et extraits des mémoires d'André Lefêure d'Ormesson, éd. A. Chéruel, Paris, Imprimerie Impériale, 1860-1861, 2 vols.

Wagner, Johann Georg, Parisische Reyss, Handlung und Pundtschwur, Solothurn, zu Johann Jacob Bernhards Truckerey, gedruckt durch Michael Wehrlin, 1664, in $4^{\circ}, 54 \mathrm{p}$.

WaSer, Johann Heinrich, Beschrybung dess Bundt Schwurs zwüschent König Ludwig XIV. zu Frankrych und Navarra und den XIII Orten auch etlichen Zugewandten loblicher Eydgnoschafft. Beschehen zu Paris im Novembri anno 1663. J. H. Waser, ZB Zentralbibliotek Zürich (Manuskripte), A 115.

\section{Travaux}

BÉLY, Lucien, «Souveraineté et souverains: la question du cérémonial dans les relations internationales à l'époque moderne", AnnuaireBulletin de la Société de l'histoire de France, Année 1993, 1994, p. 27-43. 
Borel, Tony, Une ambassade suisse à Paris: 1663: ses aventures et ses expériences, Lausanne, Payot, 1910.

Livet, Georges, Recueil des instructions aux ambassadeurs et ministres de France, tome XXX; Suisse: tome II, Paris, CNRS, 1983, 2 vols.

Maissen, Thomas, Die Geburt der Republic. Staatsverständsnis und Repräsentation in der frühneuzeitlichen Eidgenossenschaft, Göttingen, Vandenhoeck \& Ruprecht, 2006.

Marchal, Guy P., Schweizer Gebrauchsgeschichte: Geschichtsbilder, Mythenbildung und nationale Identität, Basel, Schwabe, 2006.

—, "Le rôle de la représentation symbolique dans les représentations diplomatiques: les envoyés de la Confédération helvétique à Paris", in Les Relations entre la France et les villes hanséatiques de Hambourg, Brême et Lübeck. Moyen-Age - XIX siècle, Richefort Isabelle et Schmidt Burghart (dir.),Paris/Bruxelles, P.I.E.-Peter Lang, 2006, p. 197-228.

Palmmert, Sigrid, «Kleider machen Leute, Königen machen Mode», in Beiträge zu Kunstwerken der Gottfried Keller-Stiftung: Festschrift zum 70. Geburtstag von Hanspeter Landolt, hrsg. von Johannes Stüchelberger und Christian Klemm, Berne, Bentelli, 1990, p. $49-54$.

—, "Der Allianzteppich und die Fragen von Selbstdarstellung, Repräsentation und Rezeption", in Kunst und Architektur in der Schweiz $=$ Art + Architecture en Suisse $=$ Arte + Architettura in Svizzera, Berne, Gesellschaft für Schweizerische Kunstgeschicht, 53/1 (2002), p. 51-59.

Poisson, Guillaume, "La République de Berne vue par les diplomates français" ", in Berns Goldene Zeit, hrsg. von André Holenstein, Berne, Stämpfli, 2008, p. 499.

Rотт, Edouard, Histoire de la représentation diplomatique de la France auprès des cantons suisses, de leurs alliés et de leurs confédérés. Tome III. 1663-1676, Berne, Stämpfli, 1921.

\section{Crédit iconographique}

Fig. 1: Tapisserie de l'alliance: Représentation du renouvellement de l'alliance entre Louis XIV et les envoyés de la Confédération, décrété le 18 novembre 1663. Photo Musée national suisse Zurich, dig. 4902. 
